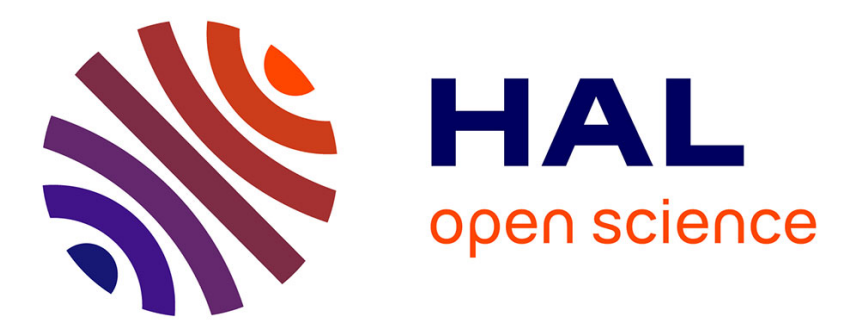

\title{
Determination of muscular fatigue in elite runners
}

Christine Hanon, Chantalle Thépaut-Mathieu, Henry Vandewalle

\section{To cite this version:}

Christine Hanon, Chantalle Thépaut-Mathieu, Henry Vandewalle. Determination of muscular fatigue in elite runners. European Journal of Applied Physiology, 2005, 94 (1-2), pp.118-125. 10.1007/s00421004-1276-1 . hal-01623765

\section{HAL Id: hal-01623765 \\ https://hal-insep.archives-ouvertes.fr/hal-01623765}

Submitted on 31 Oct 2017

HAL is a multi-disciplinary open access archive for the deposit and dissemination of scientific research documents, whether they are published or not. The documents may come from teaching and research institutions in France or abroad, or from public or private research centers.
L'archive ouverte pluridisciplinaire HAL, est destinée au dépôt et à la diffusion de documents scientifiques de niveau recherche, publiés ou non, émanant des établissements d'enseignement et de recherche français ou étrangers, des laboratoires publics ou privés. 


\section{Christine Hanon · Chantalle Thépaut-Mathieu \\ Henry Vandewalle \\ Determination of muscular fatigue in elite runners}

Accepted: 2 September 2004 / Published online: 5 February 2005

(C) Springer-Verlag 2005

\begin{abstract}
This study analyses the changes in the electromyographic activity (EMG) of six major muscles of the leg during an incremental running test carried out on a treadmill. These muscles, the gluteus maximus (GM), biceps femoris $(\mathrm{BF})$, vastus lateralis $(\mathrm{VL})$, rectus femoris $(\mathrm{RF})$, tibialis anterior (TA) and gastrocnemius $(\mathrm{Ga})$ are known to have quite different functions during running. The aim of this study was to develop a methodology adapted to the analysis of integrated EMG (iEMG) running results, and to test the chronology of the onset of fatigue of the major muscles involved in running. Nine well-trained subjects $\left[\dot{V} \mathrm{O}_{2 \max } 76\right.$ (2.9) $\left.\mathrm{ml} \cdot \mathrm{min}^{-1} \cdot \mathrm{kg}^{-1}\right]$ took part in this study. They completed a running protocol consisting of 4 min stages, incrementally increasing in speed until exhaustion. The EMG signal was recorded during ten bursts of activation analysed separately at $45 \mathrm{~s}$ and 3 min $40 \mathrm{~s}$ of each stage. During running, consideration of the alteration in stride frequency with either an increase in speed or the onset of fatigue appears to be an indispensable part of the assessment of muscular fatigue. This allows the comparison of muscular activation between the various stage speeds by the use of common working units. Distance seems to be the only working unit that allows this comparison and thus the determination of the appearance of fatigue during running. The biarticular hip-mobilising muscles (RF and BF), which present two different bursts of activation during one running cycle, are the muscles that show the earliest signs of fatigue.
\end{abstract}

C. Hanon $(\varangle) \cdot C$. Thépaut-Mathieu

Laboratoire de Biomécanique et de Physiologie,

Institut National du Sport et de l'Education Physique,

75012 Paris, France

E-mail: christine.hanon@insep.fr

Tel.: + 33-1-41744535

H. Vandewalle

Département de la Formation,

Institut National du Sport et de l'Education Physique, 75012 Paris, France
Keywords Fatigue threshold $\cdot$ Lower limbs $\cdot$ Surface electromyography $\cdot$ Treadmill running

\section{Introduction}

The existence of relationships between EMG activity and the local physiological state has incited some authors, for example Viitasalo et al. (1985) and Moritani and de Vries (1980) to examine the possibility of using EMG as a non-invasive means of assessing muscular fatigue during physical activity. Used in this sense, it provides a means of observing the appearance of fatigue at the level of the structures directly involved in driving the activity, and also for the comparison of muscles that are recruited differently. Fatigue has often been determined during incremental exercise protocols as a breakpoint in the relationship between the integrated EMG (iEMG) and exercise intensity (Nagata et al. 1981), or as a significant increase in iEMG between the beginning and the end of a given stage (Hanon et al. 1998). With this last method, it can be stated that an increase in iEMG observed at a given intensity is the result of an additional recruitment of muscle fibres.

The majority of studies in this area have been carried out using an ergocycle and have focused on the activation of the quadriceps muscles, usually the vastus lateralis (VL), vastus medialis (VM) (Viitasalo et al. 1985) or the rectus femoris (RF) (Glass et al. 1998, Lucia et al. 1998). To the best of our knowledge, only three different studies have been conducted on treadmill running (Taylor and Bronks 1994; Mateika and Duffin 1994 and Hanon et al. 1998). In these studies only the VL and/or the gastrocnemius $(\mathrm{Ga})$ were studied. No information is available concerning other muscles that have, according to Mann and Sprague (1980) and Lemaire and Robertson (1987), an important function during running such as the biceps femoris (BF), the gluteus maximus (GM) or the RF. Indeed, some muscles are monoarticular (VL, VM), whereas others are biarticular (RF, BF). 
According to Jacobs et al. (1993), monoarticular muscles are activated primarily to contribute to positive work whereas the biarticular muscles provide fine regulation of the distribution of net moments about the hip and knee joints (Ebenbichler et al. 1998).

The different muscles involved in any given activity do not have comparable modalities of activation. For example, during running, Mann et al. (1986) and Simonsen et al. (1985) have identified two distinct periods of activation for the BF as a hip extensor and knee flexor and for the RF as a hip flexor and knee extensor, and only one for the $\mathrm{Ga}$, as an ankle plantar flexor. When studying changes in muscle activation with fatigue, the characteristics of the exercise type should be taken into account. While pedalling on an ergometric cycle implies mostly a fixed cadence, running allows the participants to adjust their stride frequency and length by greater or lesser amounts (Nilsson et al. 1985). An EMG burst that depends on both the quantity and the quality of impulses can vary according to the stride frequency. Therefore, EMG burst cannot be considered as a steady parameter even during the same stage.

The present study was conducted on a treadmill, in order to test the chronology of the onset of fatigue during running by comparing muscles that have quite different functions during this activity. It was also intended to determine a method adapted to running that allows the assessment of the fatigue component in the increase in the level of muscular activation. Therefore, this study aims to consider the fact that stride frequency can change both with speed and with fatigue, which implies that this must be taken into consideration during EMG analysis. The level and duration of the muscular activation analysed per burst in the different phases of the stride should help to clarify the possibility of mechanical adaptation related to fatigue, as suggested by Williams et al. (1991).

\section{Methods}

\section{Subjects}

Nine well-trained (5-7 training sessions per week) male subjects took part in this study. Their mean (SD) age, body mass and height were 26.8 (5.13) years, 65.4 (2.20) $\mathrm{kg}$ and $179.9(3.72) \mathrm{cm}$, respectively. The subjects were informed of the experimental procedures and gave their written consent before the onset of the tests. Prior to the experiment, each subject underwent a general medical check-up.

\section{Experimental protocol}

The test included two types of exercise. An isometric protocol consisting of maximal isometric voluntary contractions of the hip extensors, hip flexors/knee extensors, knee flexors and ankle dorsal flexor was performed at the beginning of the experiment, prior the running protocol. A progressive treadmill run until exhaustion, consisting of 4-min stages separated by 1 -min rest periods was then performed.

\section{EMG data collection}

The EMG was recorded throughout the experiment (the running and isometric protocols). The EMG signals were picked up from the bellies of the VL, RF, BF, TA, and $\mathrm{Ga}$ muscles using a bipolar configuration (electrodes $10 \mathrm{~mm} \mathrm{Ag-AgCl}$, Numeris). The skin was scraped to ensure that the impedance was kept below $1,000 \Omega$. The EMG signal was recorded continuously throughout each test, amplified $(\times 600)$, filtered (bandwidth $6-1,500 \mathrm{~Hz}$, Mazet Electronic) and stored on cassette using a digital audio tape recorder (KMT D-8 Mini type, bandwidth $12 \mathrm{kHz}$ per channel with 12-bit data resolution, Sony). Off-line EMG recorded samples were digitised using a $1 \mathrm{kHz}$ sampling rate and stored on computer disk.

\section{Isometric protocol}

Several types of isometric tests were conducted, each with the assistance of a particular ergometer intended to maximally activate the desired muscle: for the VL and the RF, $120^{\circ}$ knee extensions $\left(180^{\circ}=\right.$ complete knee extension); for the $\mathrm{BF}, 150^{\circ}$ knee extensions; for the $\mathrm{GM}, 120^{\circ}$ hip extensions $\left(180^{\circ}\right.$ corresponding to alignment of trunk and leg); for the TA, $90^{\circ}$ dorsi-flexions $\left(90^{\circ}\right.$ corresponding to the anatomical angle of the leg and the foot), and for the $\mathrm{Ga}, 120^{\circ}$ plantar flexions $\left(180^{\circ}\right.$ corresponds to complete plantar flexion). The ergometers were adjusted so that the movement of the mechanical lever arm was blocked by installing a metal rod that linked the lever arm to the frame of the ergometer so that no movement was possible. A forcesensitive strain gauge was installed into this system. Except for the Ga, the desired exercise was a one-sided effort of the right member. Care was taken to align the flexion-extension axis of the joint with the axis of the ergometer and to adapt the length of the lever arm to the segment length of the subject.

Each type of maximal isometric effort was performed twice for 5-6 s, separated by 2 min of rest. During each isometric effort, the subjects were verbally encouraged. The electromyographic activity was analysed and integrated during the best $2 \mathrm{~s}$ average activation out of the two trials. This iEMG value was taken to correspond to the maximal activation ( $\mathrm{iEMG}_{\max }$ ).

\section{Running protocol}

The incremental tests were conducted on a motorised treadmill $(0 \%$ gradient $)$. The velocity was controlled continuously by an electronic cell placed on the treadmill 
belt and was increased by $2 \mathrm{~km} \cdot \mathrm{h}^{-1}$ (and by $1 \mathrm{~km} \cdot \mathrm{h}^{-1}$ from $20 \mathrm{~km} \cdot \mathrm{h}^{-1}$ ) in 4-min stages until the subjects reached exhaustion. At the end of each stage the exercise was interrupted for a 1-min rest. The test began at a treadmill velocity of $13 \mathrm{~km} \cdot \mathrm{h}^{-1}$ [corresponding to approximately $65 \%$ of the average maximal oxygen uptake $\left(\dot{V} \mathrm{O}_{2 \max }\right)$ for this group]. Before the tests, the subjects were required to run for $10 \mathrm{~min}$ at a treadmill velocity progressively increased from $9-11 \mathrm{~km} . \mathrm{h}^{-1}$ as a warm up.

\section{Determination of contact time}

In order to determine the contact time (heel touchdown to toe-off) and consequently the cycle duration (heel touchdown to heel touchdown) and flight time (toe-off to heel touchdown), an insole (Biometrics) was placed in the right shoe of each runner. This insole is composed of pressuresensitive zones. Two of these zones, corresponding to the heel and to the toe were used for this study.

\section{Run EMG analysis}

Two different methods of data analysis were utilised (Fig. 1): burst and distance analysis.

\section{EMG analysis on a burst scale}

The EMG signal recorded during the different running stages was analysed at the start (S) and at the end (E) of each 4-min stage. Ten consecutive running bursts were sampled one-by-one at $45 \mathrm{~s}$ (S) and $3 \mathrm{~min} 40 \mathrm{~s}$ (E), respectively. The corresponding EMG signal was then integrated over each whole burst to give the iEMG. The two extreme values were not taken into account and the mean value of the eight running bursts was then expressed relative to those obtained at the start of the first stage.

For biphasic muscles (RF, BF) this treatment by burst allows separate analysis of the bursts recorded during contact phase (RF-c, BF-c) and flight phase (RF$\mathrm{f}, \mathrm{BF}-\mathrm{f})$. The part of the burst recorded just before the foot contact was considered as the contact phase. The aim of this analysis was to gain information about the duration and the relative level of activation during the bursts. The duration was expressed in two ways, either in milliseconds or relative to the duration of the

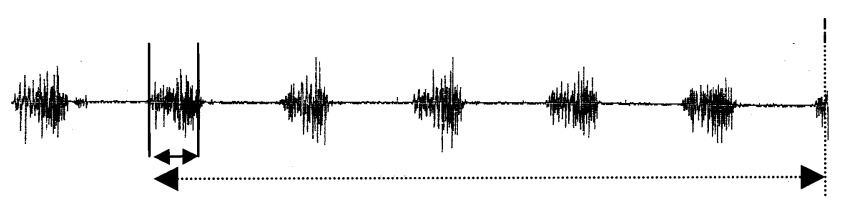

EMG analysis on burst ( -20 metres $)$ and distance scale (----) (DiEMG)

Fig. 1 EMG analysis on a burst (solid line) and the distance scale (dotted line) stride cycle. To allow comparison with the maximal muscular activation, the muscular activation produced during a burst was first expressed with regard to the burst duration (iEMG/burst duration) and then related to the $\mathrm{EEMG}_{\max }$. (see Hanon et al. 1998).

\section{EMG analysis on distance scale}

For this type of analysis, it is necessary to examine the required activations in order to produce a quantity of constant work (i.e. the body mass of the subject $x$ the movement distance). For our subjects, a distance of $20 \mathrm{~m}$ corresponded on average to 10.6 strides at $13 \mathrm{~km} \cdot \mathrm{h}^{-1}$ and 7 strides at $22 \mathrm{~km} \cdot \mathrm{h}^{-1}$.

Statistics and run-iEMG fatigue threshold determination

Changes in the data compared to changes in treadmill velocity were shown by an analysis of variance (ANOVA) for repeated measures. The run-iEMG fatigue threshold was determined by using paired Student's $t$-tests comparing the run-iEMG values between $\mathrm{S}$ and $\mathrm{E}$ of each successive stage. The first stage where a significant difference was detected was considered to be the run-iEMG fatigue threshold. Changes at a value of $P<0.05$ were considered to be significant.

\section{Results}

The physiological results [mean $\dot{V} \mathrm{O}_{2 \text { peak }} 76.1$ (2.9) $\mathrm{ml}$ $\left.\mathrm{O}_{2} \cdot \mathrm{min}^{-1} \cdot \mathrm{kg}^{-1}\right]$, and mean maximal aerobic speed [21.1 (1.2) $\mathrm{km} \cdot \mathrm{h}^{-1}$ ] are characteristic of a population of high level specialists in middle-distance running.

\section{Stride parameters}

From the beginning $\left(13 \mathrm{~km} \cdot \mathrm{h}^{-1}\right)$ to the end of the test $\left(21 \mathrm{~km} \cdot \mathrm{h}^{-1}\right)$, the stride duration, flight duration and contact duration decreased from 534 to 469,340 to 316 and 194 to $152 \mathrm{~ms}$, respectively. These differences were significant $(P<.05)$ in the middle of the tests (speeds of $\left.15-19 \mathrm{~km} \cdot \mathrm{h}^{-1}\right)$ for the stride duration, and at the end of the test $\left(19-22 \mathrm{~km} \cdot \mathrm{h}^{-1}\right)$ for the contact duration. No significant difference was noted for the flight duration.

As shown in Table 1, the stride parameter durations were steady during the major part of the test. A significant decrease was observed during the final stage for both the flight and stride durations. The contact duration remained steady throughout the test.

\section{EMG parameters}

Depending on the muscles being assessed, it was possible to observe one $(\mathrm{Ga}, \mathrm{VL})$ or two $(\mathrm{BF}, \mathrm{RF})$ bursts of 
Table 1 Evolution of the stride parameters showing the influence of speed and of stage duration. Stage $N$ Final stage, $N-1, N-2 \ldots N-4$ previous stages. $S$ Start, $E$ end

\begin{tabular}{|c|c|c|c|c|c|c|c|c|c|c|c|}
\hline & & \multicolumn{10}{|c|}{ Stages } \\
\hline & & $\mathrm{S}$ & $\mathrm{E}$ & $\mathrm{S}$ & $\mathrm{E}$ & $\mathrm{S}$ & $\mathrm{E}$ & $\mathrm{S}$ & $\mathrm{E}$ & $\mathrm{S}$ & $\mathrm{E}$ \\
\hline \multirow[t]{2}{*}{ Stride cycle duration (ms) } & $\mathrm{m}$ & 522 & 532 & 525 & 522 & 507 & 505 & 494 & 489 & $484 *$ & $461 *$ \\
\hline & ds & 23 & 26 & 25 & 28 & 30 & 24 & 29 & 28 & $12^{*}$ & $12^{*}$ \\
\hline Flight duration (ms) & $\mathrm{m}$ & 337 & 332 & 322 & 327 & 322 & 310 & 313 & 307 & $315^{*}$ & $300 *$ \\
\hline Contact duration (ms) & ds & 6 & 8 & 7 & 6 & 4 & 5 & 7 & 4 & 5 & 3 \\
\hline
\end{tabular}

$* P<0.05$ Significant difference between the start and end of the same stage

activation during each running cycle. The muscular activation for the TA appeared as a burst of activation which was not easily divisible, therefore we chose to consider it as a single burst of activation.

\section{Duration of activation}

The duration of the bursts of activation can be expressed in milliseconds or in relation to the stride duration, as discussed in the Methods section. When expressed in milliseconds, the duration of activation varied substantially among the different muscles. For instance, at $13 \mathrm{~km} \cdot \mathrm{h}^{-1}$, it increased from 111 (29) ms [burst of activation of the $\mathrm{BF}$ recorded during the flight phase (BF-f)] to 468 (53) ms (burst of activation in the TA). The duration of activation expressed in milliseconds varied during the test depending on the phase of the stride; it decreased during the contact phase when speed increases, whereas it tended to increase for bursts recorded during the flight phase (BF-f and RF-f).

When expressed relative to the stride duration, the burst durations differed considerably between the muscles; for example at $13 \mathrm{~km} . \mathrm{h}^{-1}$ the burst durations were 33.1 (5.9) \% for the VL and $86.9(6.7) \%$ for the TA. The burst durations at this time for the Ga, BF and RF were $42.4(3.7) \%, 66.8(3.4) \%$ and $39.3(7.1) \%$, respectively. The consequences of increasing speed on this parameter are quite different. The duration of activation for the $\mathrm{Ga}$ and VL tended to decrease with increases in speed $(P<0.05)$. The biarticular hip-mobilising muscles (BF, $\mathrm{RF}$ ) were activated for a longer period of time relative to the stride duration when the speed increased $[+12.98$ $(1.8) \%$ between the beginning and the end of the test, $P<0.05]$.

The duration of the burst of RF activity increased significantly during the last stage (n), from 109 (31) to 138 (25) ms for the burst of activation recorded during the contact phase (RF-c), and decreased significantly for RF-f, from 155 (31) to 138 (50) ms during the $n-2$ stage and from 157 (40) to 141 (50) ms during the $n-1$ stage.
Level of activation

At $21 \mathrm{~km} \cdot \mathrm{h}^{-1}$, the results ranged from $36 \%[\mathrm{RF}(\mathrm{c}+\mathrm{f}) / 2]$ to $62 \%[\mathrm{BF}(\mathrm{c}+\mathrm{f}) / 2]$ of maximal activation. The TA, Ga and VL were activated at 42 (8.5), 56 (7.6) and $60(82) \%$ of the $\mathrm{iEMG}_{\max }$, respectively.

\section{Determination of muscular fatigue}

On the burst scale, a significant increase in the iEMG between the start and the end of a given stage was shown for BF-c and RF-c at the end of the test. One stage before the end of the test, the level of activation for RF-f started to increase significantly $(P<0.05)$.

When analysed on the distance scale, as shown in Table 2, the Ga was the only muscle that did not show an increase in muscular activation between the $20 \mathrm{~m}$ at the start and the final $20 \mathrm{~m}$ at the end of the stage. The $\mathrm{DiEMG}_{20}$ increased significantly during the last stage for both the VL and the TA. For the BF and the RF, a significant increase in $\mathrm{iEMG}$ was observed during stages $n-2$ or $n-1$, depending on the stride phase. The chronology of the appearance of the different fatigue thresholds are presented in Fig. 2.

\section{Discussion}

The main finding of the present study is that during running the hip-mobilising muscles become fatigued earlier than the other lower limb muscles studied. These muscles show an increase in their activation in response to increases in speed. Moreover, it appears that the best methodological way to assess muscular fatigue during treadmill running is to analyse the EMG using a distance scale.

Methods of data analysis

The modes of analysis suggested in the literature concerning the appearance of fatigue during pedalling 
Table 2 Evolution of the distance integrated electromyogram (DiEMG) for the various muscles during each stage of the exercise test. Data are given as the means (SD). Ga Gastrocnemius, TA tibialis anterior, VL vastus lateralis, $B F-c$ biceps femoris, contact phase, $B F-f$ biceps femoris, flight phase, $R F-c$ rectus femoris, contact phase, $R F-f$ rectus femoris, flight phase

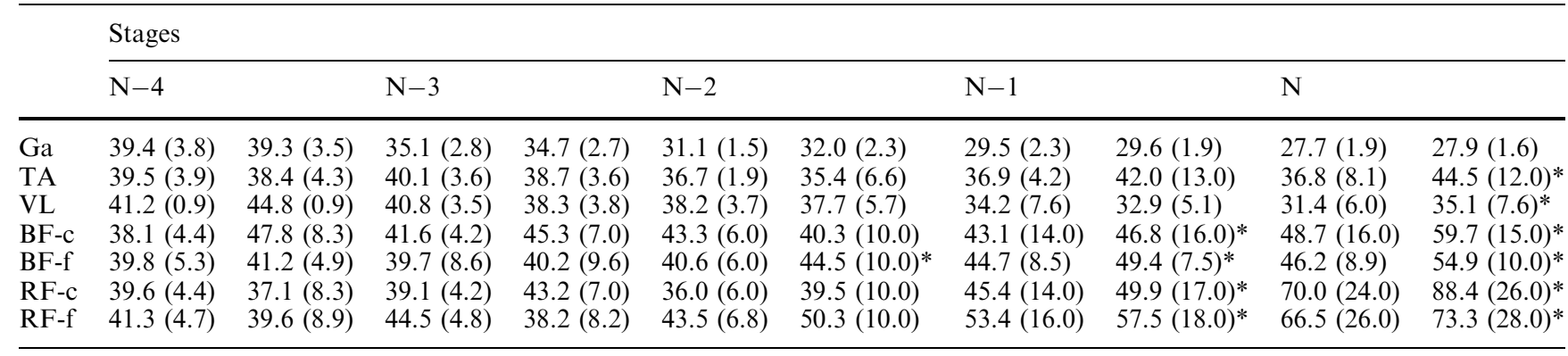

$* P<0.05$ Significant difference between the start and end of the same stage

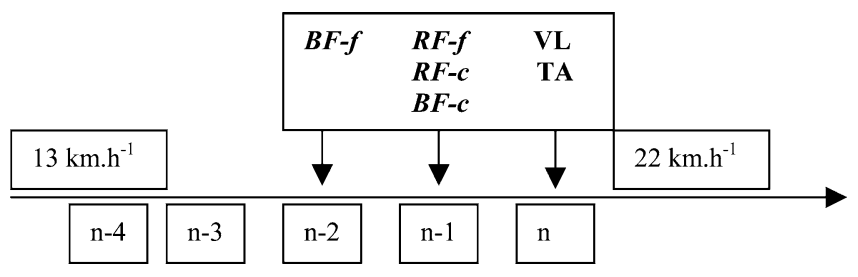

Fig. 2 Chronology of appearance of the local fatigue thresholds by distance integrated EMG. $B F$ and $R F$ hip-mobilising muscles

propose testing the evolution of the iEMG on a given number of bursts (Taylor and Bronks 1995, Viitasalo et al. 1985) or duration (Smith et al. 2000, Glass et al. 1998). These methods are not adapted to the characteristics of running. Analysis of the evolution of the stride parameters shows that on a treadmill at a given speed, the stride frequency increases with the maintenance of the last stages as has been previously observed during long-duration stages on a treadmill (Hausswirth et al. 1997, Kyrölainen et al. 2000). Thus the characteristics of each burst of activation can vary concurrently with changes in stride length and frequency. In our study, the stride frequency increased with fatigue, thus for a given number of bursts the distance covered is lower, whereas for a given duration, it can be higher. Indeed, the distance covered, and therefore the work achieved, appears to differ according to the analytical method used.

As speed is fixed and frequency increases, burst analysis can not be considered as a stable parameter. Hence, the stride length and thus the quantity of impulses during the contact phase decrease for a given stride cycle (Mero et al. 1992). The onset of fatigue, which leads to an increase in the iEMG during submaximal constant contractions (Enoka and Stuart 1992), may thus be compensated and then masked by this decrease in the number of impulses.

Averaging the level of activation (level of activation/ duration of activation) does not make it possible to solve this methodological problem. Indeed, this ratio depends on the combined influences of the increase in both the frequency and fatigue on the level and the duration of activation. Indeed, depending on the muscle, the level of activation has a tendency to increase or remain constant (VL), meaning that fatigue could have a greater influence on the level of activation than the increase in the frequency. The burst duration may remain stable $(\mathrm{Ga})$, decrease $(\mathrm{Ga}, \mathrm{TA})$ or increase (BF, RF). Thus, one can observe means of activation that evolve differently in fatigued muscles. Consideration of the mean muscular activation is relevant only if the biomechanical parameters are constant.

It therefore appeared valuable to use a work unit as a base, i.e. a common distance, for the comparison of the levels of muscular activation between the beginning and the end of a given stage. The choice of the reference distance $(20 \mathrm{~m})$ corresponds to that approximately travelled during the 10 bursts collected at $13 \mathrm{~km} . \mathrm{h}^{-1}$. At the speeds considered, the energy cost corresponding to a given distance is independent of speed (Margaria et al. 1963). Consequently, the distance covered is equivalent to the energy expenditure and can be regarded as being equivalent to the work output. The quantity of EMG per meter travelled may thus be considered as a quantity of EMG per work unit. This type of analysis involves multiplying the level of activation recorded during a burst by the number of bursts recorded over $20 \mathrm{~m}$ and comparing the values recorded at the beginning and end of the same stage. Using this method, the onset of fatigue was apparent earlier than when using the burst analysis alone. Indeed, the iEMG measured during each burst tended to increase at the end of the stage, as did the number of bursts necessary to travel the reference distance. Taking these two factors into account simultaneously accentuates the difference in muscular activation between the beginning and the end of the stage. This is particularly true for the hip-mobilising muscles (RF and BF) (Fig. 2).

Appearance of fatigue and the influence of increased speed

The RF and the BF, the two hip-mobilising muscles, were fatigued earlier than the other lower limb muscles 
studied (the VL, Ga, and TA). The RF and BF present some common characteristics; both are biarticular biphasic muscles solicited during the contact phase and the support phase, and their activation durations increase relative to the duration of the stride cycle when the speed increases. Moreover, contrary to monoarticular muscles, the level of activation recorded during a burst increased linearly with speed (Fig. 3). These activation characteristics tend to show that the increase in speed has a greater influence on these two muscles than on the other muscles tested. Our results are in accordance with previous studies (Lemaire and Robertson 1987; Belli et al. 2002) that describe the respective contribution of hip, knee and ankle muscles when speed increases. According to these authors, the role of the ankle and knee extensors is to create high joint stiffness before and during the contact phase. It is quite different to the role of the hip extensors, which are considered as the prime forward movers of the body. Moreover, changes linked to increasing running speed are noted as being particularly important at the hip joint (Belli et al. 2002). Similarly, according to Luhtanen and Komi (1978), the levels of ground reaction forces recorded on the platform are further increased with speed on the horizontal axis than on the vertical axis. Hence, one can understand why the propelling hip muscles are mobilised to a greater extent.

The role of the BF and more generally of the hip extensors in sprinting has been widely reported, in particular by Mero et al. (1992), Montgomery et al. (1994) and Simonsen et al. (1985). More recently, Kÿroläinen et al. (2001) have demonstrated an important increase in the EMG activity of the BF with increasing running speed. In the present study, the BF was activated at an average level of $62 \%$ of the $\mathrm{iEMG}_{\max }$ for $75 \%$ of the stride cycle $\left(21 \mathrm{~km} \cdot \mathrm{h}^{-1}\right)$, resulting in a recovery phase of $100 \mathrm{~ms}$, divided into two parts. The early fatigue observed for the BF in the present study can be explained by the increased activation of this muscle when speed increases.

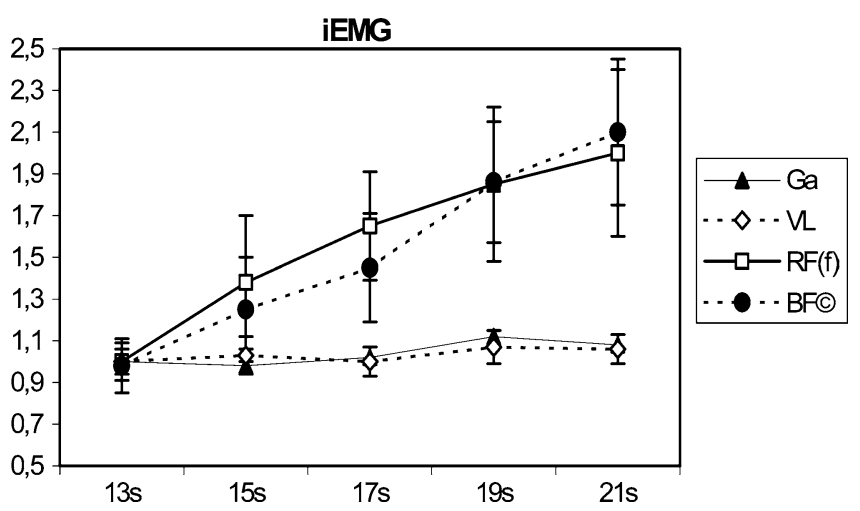

Fig. 3 Evolution of EMG activity (iEMG) with the speed of running (data are normalised according to the results of the first stage on the burst scale). Results are presented for the gastrocnemius $(G a)$, vastus lateralis $(V L)$, biceps femoris in the contact phase $(B F-c)$, and the rectus femoris in the flight phase $(R F-f)$
The early fatigue observed for the RF is more questionable; the RF appears to be recruited for an equally long period of time $\left(56 \%\right.$ of the time at $\left.21 \mathrm{~km} \cdot \mathrm{h}^{1}\right)$ but to a lower level $\left(36 \%\right.$ of $\left.\mathrm{iEMG}_{\max }\right)$ than the BF. For Williams et al. (1991) and Candau et al. (1998) some biomechanical adaptations to fatigue could result in altered activity for a particular muscle. For example, flexing the knee to a greater degree will result in a reduction of the metabolic work performed by the hip flexors as fatigue occurs (Williams 1991). Thus the increase in activation observed during the last stages for muscles like the RF that are not so intensely activated is questionable, and could equally be interpreted as compensation for fatigue of other areas.

The data of Williams et al. (1991) and Candau et al. (1998) shows a significant increase in step length with fatigue. The increase in stride length associated with fatigue as observed by these authors does not sustain our hypothesis of fatigue of the hip muscles and is contrary to our results (decrease of stride length), which argue more in favour of the hypothesis of fatigue of the RF and BF. Moreover, the level of activation of the other knee extensor assessed in the present study (VL) increased during the last stage of the running test, after the intra-stage increase of activation observed for the RF. Hence, the RF does not appear to compensate for the fatigue of the other muscles assessed in the present study.

Finally, the burst durations of the RF change during the stages depending on the cycle phase; showing an increase in RF-c during the last stage and a decrease in RF-f during $n-2$ and $n-1$. The decrease for RF-f duration seems consistent with the decrease of the duration of suspension during the last stages, but the increasing duration of activation (in the contact phase) occurs while the duration of impact is almost stable. Nevertheless, this could be either the result of fatigue (a longer duration of recruitment needed to produce the same number of impulses) or be due to biomechanical pattern changes with fatigue. It is difficult to determine if there are only quantitative changes (most importantly the number of muscular fibres recruited to limit the decline of the force during impact, an increase in the frequency of the stride to maintain the same speed) or qualitative changes, with certain muscles compensating for others.

The possibility to assess muscle outside of the running context as done by Hanon et al. (1998) and Hausswirth et al. (2000) could be an interesting methodological way to address the causes of increased activation. In these previous studies, the subjects maintained isometric contraction of the knee extensor muscles immediately after running sessions. This procedure allowed freedom from modifications of biomechanical patterns. Unfortunately, it is not possible to test more than one muscle just after the end of a running session and the muscle assessed in these previous studies was the VL. In future studies, this methodological approach could be used for the RF. 
Causes of fatigue

Nevertheless, our study presents more arguments in favour of fatigue of the RF being observed in the two last stages than in favour of biomechanical adaptations. The influence of speed is particularly important for this muscle. The inter-stage iEMG increase observed during the suspension phase corresponds to the shift in activation of the RF noted by Nilsson et al. (1985). Moreover, it has also been noted by many authors, including Eldgerton et al. (1975), that the RF is a muscle characterised by a typology strongly dominated by type II fibres, which according to Komi and Tesch (1979) among others, makes it a fatigable muscle.

The early intra-stage increase in activation developed by the RF emphasises the influence of the frequency of initiation of contractions on the onset of fatigue. This phenomenon has already been observed by Bergstrom and Hultman (1988) who compared two continuous and intermittent work regimes and showed that fatigue develops faster in the case of an intermittent regime. Duchateau and Hainaut (1985) have argued that to develop repetitive muscular tension costs more than to maintain it, principally because the $\mathrm{Ca}^{2+}$ ATPase of the sarcoplasmic reticulum must restore the intracellular gradients to the rest state after each contraction.

The early onset of fatigue for the BF and RF, the two biphasic muscles, also leads us to think that the ratio of working time/rest period, defined by Green (1991) as the 'duty cycle' can be called upon. Indeed, the quality of irrigation of these muscles and thus the $\mathrm{O}_{2}$ supply as well as the release of the metabolites is deteriorated by an important duty-cycle (Hermansen 1981). It was shown that during running the muscles investigated are subjected to an alternation of working time/rest period which varies greatly, according to their role during running. This ratio makes it possible to at least partly explain the chronology of the onset of fatigue during running; the biarticular hip-mobilising muscles (the $\mathrm{BF}$ and RF) are the first muscles to fatigue whereas the Ga does not present any sign of fatigue in this study. Moreover, as observed by Kyröläinen at al. (2000), the increased stride frequency may have increased the amount of muscular activity by shortening the duration of relaxation.

In conclusion, it appears that the best methodological way to assess muscular fatigue during running on a treadmill is to analyse the EMG according to a distance scale. Our results reveal that the hip-mobilising muscles are fatigued earlier than the other lower limb muscles studied in this study. It seems reasonable to suggest the hypothesis that the clear increase in the participation of the $\mathrm{BF}$ and the RF to the high speed noted here (and which is well supported by the literature) is consistent with the linear increase of the stride length with speed, and explains the resulting fatigue. Possibly due to the short duration of the stages used in our protocol (4 min), biomechanical adaptations to fatigue were not clearly demonstrated in this study.
Nevertheless, we can hypothesise that depending on the speed, when exercise continues, the muscles exhausted earliest may be different: the Ga, and the VL at lower speeds; the BF and the RF at higher speeds. In the first case, the duration of the stages should be longer than in the present study to evidence fatigue of the $\mathrm{Ga}$ and VL. In addition, concerning running, two types of adaptation to fatigue should be differentiated depending on whether the context allows (track) or not (treadmill) a decrease in speed.

Acknowledgements The authors would like to thank Niamh Kelly and Kristen Neymarc for proof-reading this article.

\section{References}

Belli A, Kyrölainen H, Komi PV (2002) Moment and power of lower limb joints in running. Int J Sports Med 23:136-141

Bergstrom M, Hultman E (1988) Energy cost and fatigue during intermittent electrical stimulation of human skeletal muscle. J Appl Physiol 65:1500-1505

Candau R, Belli A, Millet GY, Georges D, Barbier B, Rouillon JD (1998) Energy cost and running mechanics during a treadmill run to voluntary exhaustion in humans Eur J Appl Physiol 77:479-485

Duchateau J, Hainaut K (1985) Electrical and mechanical failures during sustained and intermittent contractions in humans. J Appl Physiol 58:942-947

Ebenbichler G, Kollmitzer J, Glocker L, Bogdansky T, Kopf A, Fialka V (1998) The role of the biarticular agonist and cocontracting antagonist pair in isometric muscle fatigue. Muscle Nerve 21:1706-1713

Eldgerton V, Smith J, Simpson D (1975) Muscle fiber type populations of human leg muscles. Histochem J 7:259-266

Enoka RM, Stuart DG (1992) Neurobiology of muscle fatigue. J Appl Physiol 72:1631-1648

Glass SC, Knowlton RG, Sanjabi PB, Sullivan JJ (1998) Identifying the integrated electromyographic threshold using different muscles during incremental cycling exercise. J Sports Med Phys Fitness 38:47-52

Green HJ (1991) Muscle power: fibre type, recruitment, metabolism and fatigue. In: Jones NL, Mc Cartney N, Mc Comas AJ (eds) Human muscle power. Masson, Paris, pp 63-79

Hanon C, Thépaut-Mathieu C, Hausswirth C, Le Chevalier JM (1998) The electromyogram as an indicator of neuromuscular fatigue during incremental exercise. Eur J Appl Physiol 78:315323

Hausswirth C, Brisswalter J, Vallier JM, Smith D, Lepers R (2000) Evolution of electromyographic signal, running economy, and perceived exertion during different prolonged exercises. Int J Sports Med 21:429-436

Hermansen L (1981) Effect of metabolic changes on force generation in skeletal muscle during maximal exercise. In: Porter R, Whelan $\mathbf{J}$ (eds) Human muscle fatigue: physiological mechanisms. Pitman Medical, London

Jacobs R, Bobbert MF, Van Ingen Schenau GJ (1993) Function of mono- and biarticular muscles in running. Med Sci Sports Exerc 25:1163-1173

Kyröläinen H, Pullinen T, Candau R, Avela J, Huttunen P, Komi PV (2000) Effects of marathon running on running economy and kinematics Eur J Appl Physiol 82:297-304

Kyröläinen H, Belli A, Komi PV (2001) Biomechanical factors affecting running economy. Med Sci Sports Exerc 33:1330-1337

Komi PV, Tesch P (1979) EMG frequency spectrum, muscle structure and fatigue during dynamic contractions in man. Eur J Appl Physiol 42:41-50

Lemaire ED, Robertson DGE (1989) Power in sprinting. Track Field J 35:13-17 
Lucia A, Pardo J, Durantez A, Hoyos J, Chicharro JL (1998) Physiological differencies between between elite and professional road cyclists. Int J Sports Med 19:342-348

Luhtanen P, Komi PV (1978) Mechanical energy states during running. Eur J Appl Physiol Occup Physiol 38:41-48

Mann RA, Sprague P (1980) A kinetic analysis of the ground leg during sprint running. Res Q Exerc Sport 51:334-348

Mann RA, Moran G, Dougherty S (1986) Comparative electromyography of the lower extremity in jogging, running, and sprinting. Am J Sports Med 14:501-510

Margaria R, Cerretelli P, Aghemo P, Sassi G (1963) Energy cost of running. J Appl Physiol 18:367-370

Mateika JH, Duffin J (1994) Coincidental changes in ventilation and electromyographic activity during consecutive incremental exercise tests. Eur J Appl Physiol 68:54-61

Mero A, Komi PV, Gregor R (1992) Biomechanics of sprint running. Sports Med 13:376-392

Montgomery WH, Pink M, Perry J (1994) Electromyographic analysis of hip and knee musculature during running. Am J Sports Med 22:272-278

Moritani T, de Vries HA (1980) Anaerobic threshold determination by surface electromyography. Med Sci Sports Exerc 12:86

Nagata A, Muro M, Moritani T, Yoshida T (1981) Anaerobic threshold determination by blood lacate and myoelectric signals. Jpn J Physiol 31:585-597
Nilsson J, Thorstensson AJ, Halbertsma J (1985) Changes in leg movements and muscle activity with speed of locomotion and mode of progression in humans. Acta Physiol Scand 123:457475

Simonsen E, Thomsen L, Klausen J (1985) Activity of mono-and biarticular leg muscles during sprint running. J Appl Physiol 54:524-532

Smith NA, Kerr GK, Green S (2000) Myoelectric patterns constant load cycle ergometer exercise. Fifth annual congress of the European college of sport science, Jyvaskyla, Finland, 19-23 July 2000, p 604

Taylor AD, Bronks R (1994) Electromyographic correlates of the transition from aerobic to anaerobic metabolism in treadmill running. Eur J Appl Physiol 69:508-515

Taylor AD, Bronks R (1995) Reproducibility and validity of the quadriceps muscle integrated electromyogram threshold during incremental cycle ergometry. Eur J Appl Physiol 70:252-257

Viitasalo JT, Luhtanen P, Rahkila P, Rusko H (1985) Electromyographic activity related to aerobic and anaerobic threshold in ergometer bicycling. Acta Physiol Scand 124:287-293

Williams KR, Snow R, Agruss C (1991) Changes in distance running kinematics with fatigue Int J Sport Biomechan 7:138162 